\title{
Review
}

\section{New Drugs Bringing New Challenges to AML: A Brief Review}

\author{
Zhi Han Yeoh ${ }^{1}$, Ashish Bajel ${ }^{1,2}$ and Andrew H. Wei ${ }^{1,3,4, *}$ \\ 1 Department of Clinical Haematology, Peter MacCallum Cancer Centre \& The Royal Melbourne Hospital, \\ Melbourne, VIC 3000, Australia; zhihan.yeoh@petermac.org (Z.H.Y.); ashish.bajel@mh.org.au (A.B.) \\ 2 The Sir Peter MacCallum Department of Oncology, University of Melbourne, Melbourne, VIC 3000, Australia \\ 3 Department of Clinical Haematology, Alfred Hospital and Monash University, \\ Melbourne, VIC 3004, Australia \\ 4 Division of Blood Cells and Blood Cancers, Walter and Eliza Hall Institute of Medical Research, \\ Melbourne, VIC 3052, Australia \\ * Correspondence: andrew.wei@monash.edu
}

check for

updates

Citation: Yeoh, Z.H.; Bajel, A.; Wei, A.H. New Drugs Bringing New Challenges to AML: A Brief Review. $J$. Pers. Med. 2021, 11, 1003. https:// doi.org/10.3390/jpm11101003

Academic Editor: Stephen Opat

Received: 5 September 2021

Accepted: 1 October 2021

Published: 3 October 2021

Publisher's Note: MDPI stays neutral with regard to jurisdictional claims in published maps and institutional affiliations.

\begin{abstract}
The better understanding of the genomic landscape in acute myeloid leukaemia (AML) has progressively paved the way for precision medicine in AML. There is a growing number of drugs with novel mechanisms of action and unique side-effect profiles. This review examines the impact of evolving novel therapies on survival in AML and the challenges that ensue.
\end{abstract}

Keywords: AML; survival; precision medicine; FLT3; IDH; TP53

\section{Introduction}

The last few years have witnessed a therapeutic renaissance in the field of acute myeloid leukemia (AML), spearheaded by the FDA's approval of 10 new therapies since 2017. While treatment for AML remains a therapeutic challenge, new therapeutic options have begun to create an emerging precision medicine framework in our approach to managing this condition. Along with the growing number of drugs with novel mechanisms of action is the arrival of an array of new adverse event profiles that are often uniquely linked to a particular class of drugs. This review examines the impact of evolving novel therapies on survival in AML and some of the novel toxicities associated with their introduction. We also discuss emerging strategies used to augment and further improve efficacy and the role of measurable residual disease (MRD) as a means for the assessment of clinical outcomes. We propose that the current evidence points to a future of AML management that will be more personalized in terms of treatment selection, safety planning, monitoring for disease response, and the re-evaluation of the mechanisms of adaptive resistance at relapse.

\section{Targeting Mutated Proteins}

\subsection{FLT3}

FLT3 mutations are one of the most common and prognostically important genetic alterations that occur in AML. FLT3 mutations are present in approximately $30 \%$ of all patients with AML, with internal tandem duplication (ITD) accounting for approximately $25 \%$ of all AML cases and FLT3 mutation in the tyrosine kinase domain (FLT3-TKD) accounting for approximately $7-10 \%$ of all cases. Both mutations lead to the constitutive activation of the receptor and its downstream signalling pathways, including PI3K/AKT/mTOR, RAS/MAPK, and STAT5 (Figure 1). FLT3 mutations cause leukaemic blast survival and proliferation [1]. FLT3-ITD mutations in AML are known unfavorable prognostic markers, as they confer poor overall survival (OS) and relapse-free survival [2]. 


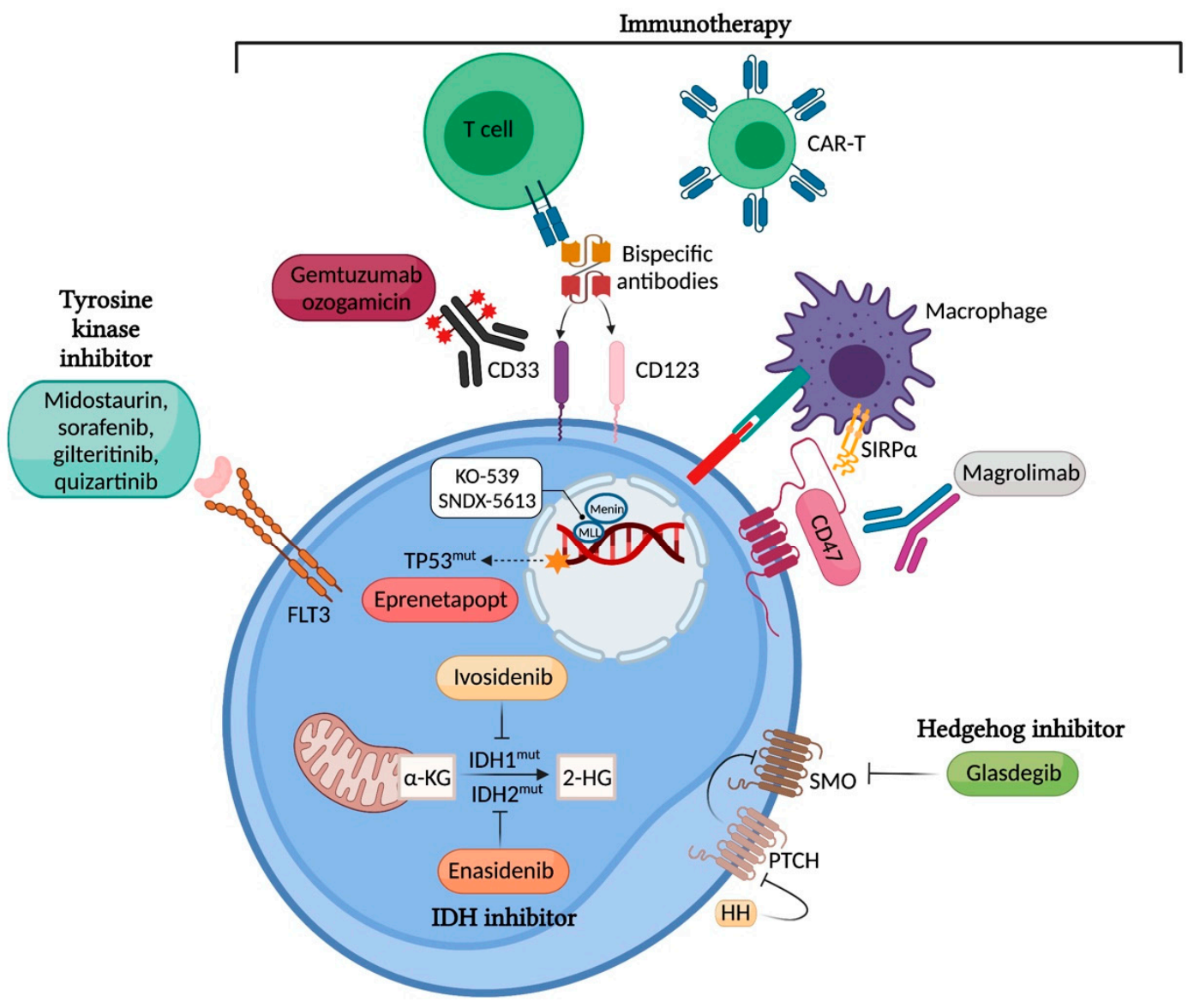

Figure 1. Targeting mutated proteins in AML. Image created with BioRender.com [3] (accessed on 26 September 2021).

Targeted therapy with FLT3 inhibitors has significantly improved outcomes in this subgroup. First-generation tyrosine kinase inhibitors (TKIs), such as midostaurin, sorafenib, and lestaurtinib, have demonstrated limited anti-leukemic activity as monotherapies. Midostaurin was shown to improve 4 -year OS from $44 \%$ to $51 \%$ when combined with intensive chemotherapy in patients with untreated FLT3-mutated AML, as demonstrated in a landmark randomized phase 3 study (RATIFY) [4]. Consequently, midostaurin had been approved as first-line therapy for FLT3 mutant AML in combination with intensive induction and consolidation therapy; it has also been approved for use as a single-agent maintenance therapy in some countries. Another first-generation TKI sorafenib, in combination with intensive chemotherapy, has been shown to have anti-leukemic efficacy, with a 2 -year event-free survival of 40-60\% [5,6]. A recent randomized study reported a relapsefree survival of $61 \%$ in the sorafenib group compared to $36 \%$ in the placebo group after achieving complete remission [7]. The current standard of care for eligible patients with FLT3-mutant AML is frontline midostaurin in combination with intensive chemotherapy and consideration for allogeneic haematopoietic stem cell transplant (alloHSCT) in first remission, given the high relapse rates after traditional cytotoxic chemotherapy.

New data concerning the role of other FLT3 inhibitors for the first-line treatment of patients fit for intensive chemotherapy are emerging. The Australasian Leukaemia and Lymphoma Group (ALLG) conducted a randomized, placebo-controlled phase 2 trial of sorafenib in combination with intensive induction and consolidation chemotherapy followed by 12 months of sorafenib maintenance. Several themes emerged, including the high rate of clinical response in both the sorafenib and placebo arms (91\% vs. $94 \%)$; the higher rate of early FLT3-ITD MRD clearance, as assessed by next-generation sequencing (NGS) in the sorafenib arm ( $43 \%$ vs. $32 \%)$; the high rate of alloHSCT in both arms ( $60 \%)$; and the high frequency of FLT3-ITD-negative relapse $(\sim 60 \%)$ [7]. Although the outcomes were similar to 
those of prior sorafenib single-arm studies [8], this study did not demonstrate a significant improvement in either EFS or OS for the sorafenib arm. A sub-group analysis showed a trend for improved OS amongst patients with a higher FLT3-ITD allelic ratio $(>0.7)$, which is consistent with the potentially greater oncogenic dependency on FLT3 in this patient population [7]

In the frontline treatment setting, gilteritinib in combination with chemotherapy has also shown favorable anti-leukemic responses, with a composite $\mathrm{CR}$ of $\sim 80 \%$ and a 70\% mutational clearance of FLT3-ITD found in a Phase I study [9]. Based on these results, randomized trials of induction and consolidation chemotherapy plus gilteritinib vs. midostaurin in FLT3-mutant AML patients are underway (NCT04027309) (Table 1). Similar phase III studies of quizartinib vs. placebo (NCT02668653) and crenolanib vs. midostaurin (NCT03258931) in combination with chemotherapy are also ongoing, aiming to provide evidence on the most efficacious FLT3 TKI in newly diagnosed FLT3-mutant AML. In patients unfit for intensive chemotherapy, azacitidine and gilteritinib have yielded promising preliminary results with a $67 \% \mathrm{CR}(\mathrm{n}=15)$ [10]. Further results of randomized studies are awaited before this combination can be incorporated as standard-of-care practice in the real world.

Table 1. Randomized clinical trials for drug development in AML.

\begin{tabular}{|c|c|c|c|}
\hline Class of Drugs & $\begin{array}{l}\text { Investigated } \\
\text { Agent }\end{array}$ & Investigation & $\begin{array}{l}\text { Trial Registration } \\
\text { Number }\end{array}$ \\
\hline \multirow{5}{*}{ FLT3 inhibitors } & \multirow{3}{*}{ Gilteritinib } & $\begin{array}{l}\text { Phase III, frontline, gilteritinib vs. midostaurin in combination with } \\
\qquad 7+3 \text { ", FLT3 mut AML }\end{array}$ & NCT04027309 \\
\hline & & $\begin{array}{l}\text { Phase III, maintenance, gilteritinib vs. placebo, FLT3 mut AML in } \\
\text { CR1 after chemotherapy }\end{array}$ & NCT02927262 \\
\hline & & $\begin{array}{l}\text { Phase III, maintenance, gilteritinib vs. placebo, FLT3 mut AML } \\
\text { after allogeneic haematopoietic stem cell transplantation }\end{array}$ & NCT02997202 \\
\hline & Quizartinib & $\begin{array}{l}\text { Phase III, frontline, quizartinib vs. placebo in combination with "7+ } \\
\text { 3", FLT3 mut AML }\end{array}$ & NCT02668653 \\
\hline & Crenolanib & $\begin{array}{l}\text { Phase III, frontline, crenolanib vs. midostaurin in combination with } \\
\qquad 7+3 \text { ", FLT3 mut AML }\end{array}$ & NCT03258931 \\
\hline \multirow[t]{2}{*}{ IDH1 inhibitors } & \multirow[t]{2}{*}{ Ivosidenib } & $\begin{array}{l}\text { Phase III, frontline, ivosidenib vs. placebo in combination with "7+ } \\
\text { 3", IDH1 mut AML }\end{array}$ & NCT03839771 \\
\hline & & $\begin{array}{l}\text { Phase III, frontline, ivosidenib vs. placebo in combination with } \\
\text { azacitidine, IDH1 mut AML }\end{array}$ & NCT03173248 \\
\hline \multirow[t]{2}{*}{ IDH2 inhibitor } & \multirow[t]{2}{*}{ Enasidenib } & $\begin{array}{l}\text { Phase III, frontline, enasidenib vs. placebo in combination with "7 + } \\
\text { 3", IDH2 mut AML }\end{array}$ & NCT03839771 \\
\hline & & $\begin{array}{l}\text { Phase III, enasidenib vs. conventional care, } \geq 60 \text { years, late stage } \\
\text { IDH } 2 \text { mut AML }\end{array}$ & NCT02577406 \\
\hline $\begin{array}{l}\text { Hedgehog } \\
\text { inhibitor }\end{array}$ & Glasdegib & $\begin{array}{l}\text { Phase III, frontline, glasdegib vs. placebo in combination with "7+ } \\
\text { 3" or azacitidine, fit and unfit patients }\end{array}$ & NCT03416179 \\
\hline
\end{tabular}

Abbreviation: " $7+3$ ", intensive induction chemotherapy with 7 days of cytarabine and 3 days of anthracycline.

Despite the improved OS and EFS found in the RATIFY trial, only 59\% of patients in the midostaurin arm achieved CR and almost half of these patients relapsed after achieving remission. AlloHSCT remains beneficial for sustaining long-term remission [11]. A post hoc analysis of RATIFY confirmed that midostaurin conferred survival benefits in FLT3-ITD AML across all three ELN risk groups, irrespective of the FLT3-ITD allelic burden or co-mutation profile (e.g., with or without NPM1 mutation) [11]. The similarly improved outcomes among patients with a low FLT3-ITD allelic burden suggest that some of the clinical benefits associated with this multikinase inhibitor may be mediated through actions targeting other anti-leukemic pathways [11]. The SORAML trial examined the role of combining sorafenib with intensive chemotherapy in patients with AML, not limited to 
those with the FLT3 mutation. This study demonstrated improved EFS outcomes across a diverse AML cohort, supporting the potential for a biologically relevant off-target activity associated with multikinase inhibitors in combination with chemotherapy in the first line treatment of AML [12].

There is a need for increased awareness of how to anticipate, mitigate, and manage complications associated with the increasing use of FLT3 inhibitors. Severe adverse events associated with the use of multikinase FLT3 inhibitors include midostaurin-related grade 3 to 5 pneumonitis or radiographic pulmonary opacities, which were reported in $8 \%$ of patients in the phase RATIFY trial [2]. Among older populations ( $>60$ years) receiving midostaurin in combination with intensive chemotherapy, the risk of cardiac toxicity was reported in $22 \%$ of patients, highlighting the importance of ECG and electrolyte monitoring in this patient population. Older patients also experienced more pulmonary adverse events than younger patients did (14\% vs. $7 \% ; p=0.07)$ [13].

In relapses after first-line midostaurin in combination with intensive chemotherapy, different modes of treatment failure have also been noted. A recent study by Schmalbrock et al. provided novel insights into clonal evolution and resistance mechanisms in the RATIFY study. Almost half the midostaurin-treated patients (46\%) were FLT3-ITD-negative at the time of disease relapse or progression [14]. In other patients, FLT3 selective pressure propagated mutations in the MAPK pathway, potentially bypassing the effect of FLT3 inhibition [14].

In relapsed refractory $(R / R)$ AML, second-generation TKIs including quizartinib, crenolanib, and gilteritinib have shown favorable single-agent clinical efficacy, related in part to their enhanced FLT3 target affinity. Gilteritinib was shown to be superior to salvage chemotherapy in the $R / R$ setting by improving the median OS from 5.6 to 9.3 months $(p=0.007)$ in the ADMIRAL study [4]. Gilteritinib is now considered as the treatment of choice for patients with R/R FLT3-mutant AML at first salvage. Adaptive resistance to gilteritinib includes off-target mutations involving the RAS-MAPK pathway and on-target drug resistant FLT3 mutations. In many cases, the adaptive resistance was polyclonal. Furthermore, in approximately half of the relapsing cases, the cause of treatment failure was unknown, indicating that other mechanisms remain to be identified.

Given the clinical limitations of monotherapy, TKI combination approaches have received considerable research interest. A recent Phase I study found that the combination of gilteritinib and venetoclax in R/R FLT3-mutant AML achieved a modified composite $\mathrm{CR}(\mathrm{CR}+\mathrm{CRp}+\mathrm{CRi}+\mathrm{MLFS})$ of $76.4 \%$, including a significant proportion (62.5\%) of patients with prior FLT3 TKI exposure, in contrast to $12 \%$ in the ADMIRAL study [15]. This suggests the promising anti-leukemic activity of this combination compared to singleagent gilteritinib with the vigilant management of cytopenias using dose interruption and modification [15].

Following the attainment of remission, FLT3 TKIs have been actively explored as maintenance therapies for patients with FLT3-mutant AML. However, the impact of maintenance therapy on survival is yet to be ascertained. The beneficial effects of sorafenib maintenance after alloHSCT have been demonstrated based on two randomized trials (NCT02156297, NCT02474290), albeit most patients were not exposed to FLT3 TKIs prior to alloHSCT $[16,17]$. A systematic review of seven studies (five sorafenib studies and two midostaurin studies) suggested that TKI maintenance therapy after alloHSCT was associated with a marked $65 \%$ reduced risk of relapse, as well as improved RFS and OS [18]. These apparent survival and relapse benefits came at the expense of the possible increased risk of acute and chronic graft versus host disease with sorafenib [18]. Prospective randomized trials testing the efficacy of gilteritinib maintenance, following consolidation (NCT02927262), and after alloHSCT (NCT02997202) (Table 1) remain critical to determine the ultimate role of FLT3 TKIs in maintenance therapies and the necessary treatment duration for FLT3-mutant AML. Future studies are required to identify the ideal TKI in terms of efficacy and safety in the post-transplant setting. 


\subsection{IDH1 and IDH2}

Isocitrate dehydrogenases (IDHs) are NADP-dependent enzymes involved in cellular energy generation in the Krebs cycle. IDH catalyzes the oxidative decarboxylation of isocitrate to $\alpha$-ketoglutarate $(\alpha-K G)$. Somatic gain-of function mutations in IDH1 or IDH2 are found in approximately $20 \%$ of newly diagnosed AML, with a higher frequency seen in older patients. Mutant forms of IDH1 and IDH2 gain neomorphic function and convert $\alpha$ KG to oncometabolite 2-hydroxyglutate, which in turn inhibits $\alpha$-KG-dependent enzymes (Figure 1). This results in pro-leukemic epigenetic changes, including the disruption of TET2 function and the suppression of granulocyte maturation [19-21].

Ivosidenib and enasidenib, selective inhibitors of mutant IDH1 and IDH2, respectively, have demonstrated encouraging clinical activity for use as monotherapies in the $R / R$ and, more recently, in the frontline setting. In patients with R/R IDH1/2-mutant AML, ivosidenib and enasidenib have induced therapeutic responses in a considerable proportion of patients with an ORR $\sim 40 \%$, CR rates $\sim 20 \%$, and median time to CR of approximately 3 months [22,23]. More recently, Roboz et al. demonstrated that ivosidenib induced deep and durable remissions in treatment-naïve IDH1-mutant AML, with a CR/CRh rate of $42.4 \%$, a mutation clearance in $64 \%$, and in some cases $(5 / 33)$ a durable remission beyond 2 years [24]. Similar observations have been noted in IDH2-mutant AML using enasidenib with a CR/CRi 30.8\% [25]. The median OS achieved with targeted monotherapy in IDH1- and IDH2-mutant untreated AML-12.6 months and 11.3 months, respectivelyis favorable compared to that of azacitidine (10.3 months) and decitabine (7.7 months) in a similar patient population [24-27].

Despite the promising results of early-phase studies, comparative randomized trials with IDH inhibitors have delivered mixed results. The phase III IDHENTIFY trial was planned as the main study to obtain full registration for the IDH2 inhibitor enasidenib. However, this was to no avail, as the study did not demonstrate a superior OS as anticipated. IDHENTIFY was an open-label trial for patients aged $\geq 60$ years who had received 2-3 prior AML therapies. Investigators preselected patients to one of four conventional care regimens $(C C R)$ in the control arm, including either azacitidine, intermediate-dose cytarabine, lowdose cytarabine (LDAC), or best supportive care. Despite the improved ORR found in the Enasidenib arm $(40.5 \%$ vs. $9.9 \%)$, the primary endpoint of OS was not significantly different between the Enasidenib and CCR arms: 6.5 months vs. 6.2 months (HR 0.86; $p=$ 0.23) [28]. The study outcome was partially compromised by $12 \%$ of patients in the CCR arm receiving post-study enasidenib [28]. Furthermore, the response and potential benefit from enasidenib are likely lower among patients with more advanced disease. In IDH2mutant AML, the combination of azacitidine and enasidenib vs. azacitidine monotherapy in a phase II study resulted in a significant improvement in response rates (ORR 71\% vs. $42 \%, p<0.01$; CR $53 \%$ vs. $12 \%, p<0.01$ ) and durations (median DOR 24.1 months vs. 12.1 months, $p=0.05$ ) [29]. Again, the survival outcome was compromised by a $21 \%$ post-study crossover in the azacitidine arm to enasidenib [29]. This reflects lessons learned from the design of phase III studies; future studies will therefore aim to focus more on the role of IDH inhibitors at earlier stages of disease and in combination with other drugs.

Among older patients unfit for intensive chemotherapy, the combination of azacitidine and ivosidenib in a phase Ib study demonstrated an ORR of $78.3 \%$, including CR in $60.9 \%$ and mutation clearance in $43.4 \%$ [30]. The median duration of response (DoR) in responders was not reached, with median follow-up of 16 months [30]. A phase III AGILE study (NCT03173248) to examine the efficacy of azacytidine and ivosidenib vs. azacitidine only in the frontline setting was stopped early due to the compelling improvement for enhanced outcomes in the ivosidenib arm, including improved EFS, the primary endpoint of the trial, as well as improved overall survival and clinical response [31].

Although ivosidenib and enasidenib responses are clinically durable, the complex genetic heterogeneity of AML suggests that combination with other non-targeted therapies may augment remission and overcome primary resistance [32]. Promising preliminary efficacy supports the combination of ivosidenib or enasidenib with intensive chemotherapy 
(Table 1 ) in newly diagnosed AML, with composite CR rates (CR + CRi + CRp) of $77 \%$ in the ivosidenib group and $74 \%$ in the enasidenib group; mutation clearance was also observed in $39 \%$ and $23 \%$ patients with the IDH1 and IDH2 mutations, respectively [33]. The clearance of FLT3 and RAS mutations in this study supports the role of combining IDH inhibitors with intensive chemotherapy to limit primary resistance and relapses mediated by signaling mutations [33].

The emerging role of IDH inhibitors in AML has also led to improved insights into the mechanisms of therapeutic resistance to these agents. An analysis of 179 patients with $R / R$ AML treated with ivosidenib suggested that primary resistance to ivosidenib was related to receptor tyrosine kinase (RTK) pathway mutations such as NRAS and PTPN11 [34]. It was also proposed that acquired resistance at disease relapse after initial response was caused by the outgrowth of off-target clones with the activation of the RTK pathway, as well as the acquisition of 2-HG-restoring mutations, including novel second-site allosteric mutations at the IDH protein-dimer interface either in the mutated IDH gene (mutation in cis) or in the other wild-type IDH1/IDH2 allele (mutation in trans) interrupting the binding efficiency of IDH inhibitor to its cognate binding site [34,35]. Although reductions in the IDH variant allele frequency and molecular clearance were associated with achieving CR, the ability to respond to IDH inhibitors did not correlate with IDH mutation burden [32]. One hypothesis is that targeting mutant IDH cells quenches the effect of the oncometabolite 2-HG from exerting a paracrine effect on neighbouring non-IDH mutant blasts. The biomarkers associated with clinical response remain unclear. In examining the prognostic significance of co-mutations in IDH-mutant AML, the NPM1 mutation was associated with improved OS in IDH1- or IDH2R140-mutated AML treated with intensive chemotherapy, suggesting a potential group of patients who would particularly benefit from the addition of targeted therapy [36].

BCL2 signaling is thought to be a critical survival pathway in IDH1/IDH2-mutant AML based on preclinical studies, providing a rationale for targeted BCL2 inhibition in IDHmutant AML [37]. Notably, a particularly favorable response was seen in the subgroup of patients with IDH-mutant AML using a combination of BCL2 inhibitor venetoclax and azacitidine (12-month OS $66.8 \%$ in venetoclax and azacitidine versus $35.7 \%$ in the azacitidine only group) [38].

As IDH inhibitors restore myeloid differentiation, they can induce myeloid maturation and proliferation, resulting in IDH inhibitor "differentiation syndrome". This is reported in $\sim 15 \%$ patients and may occur with hyperleucocytosis [24,25]. Often occurring during the initial period of myeloid maturation, it is a non-specific syndrome manifesting as dyspnea, hypoxia, culture-negative fever, pulmonary infiltrates, pleural or pericardial effusions, peripheral edema, and weight gain. A high clinical index of suspicion is required for the timely initiation of corticosteroids, as these symptoms frequently overlap with infections and progressive AML.

\subsection{TP53}

In AML, the TP53 mutation accounts for approximately $5-20 \%$ of cases. It is associated with a complex and/or monosomal karyotype with very poor survival outcomes after conventional treatment options, with reported CR rates of $20 \%$ using hypomethylating agents and a median OS of only 6-12 months [39,40]. TP53 functions as a tumor suppressor gene and the majority of TP53 mutations are missense in nature and associated with dominant negative function [41].

Following the recent discovery that TP53 mutations in myeloid malignancies drive clonal selection through a dominant-negative effect without gain-of-function activities, several novel small molecules to reactivate missense-mutant p53 protein have been investigated to restore the wild-type function of p53 (Figure 1) [41-43]. Of these, eprenetapopt (APR-246), a first-in-class mutant p53 reactivator, has received considerable attention due to its early but compelling clinical efficacy when combined with azacitidine in TP53-mutant MDS and AML [43-45]. It is a prodrug that is spontaneously converted to active substance 
methylene quinuclidinone (MQ) under physiological conditions and binds covalently to specific cysteine residues in the mutant p53 to induce apoptosis [42].

A recent phase II study (NCT03072043) of azacitidine and eprenatopopt reported an ORR of $73 \%$, with $50 \%$ achieving CR in the MDS cohort $(n=40)$ and an ORR of $64 \%$, with $36 \%$ achieving CR in the oligoblastic AML cohort $(n=11)$ [44]. The median OS was 10.8 months, with significant survival improvement seen in the responders (14.6 vs. 7.5 months) [44]. Comparable rates of clinical response were also demonstrated in another phase II study by Groupe Francophone des Myélodysplasies (GFM) (NCT03588078), with an ORR 63\% (47\% CR) in MDS and an ORR 33\% (17\% CR) in oligoblastic AML. Although there were promising phase II results in both AML and MDS, the phase III study in MDS (NCT03745716) did not demonstrate a tbetter CR rate. Phase 3 results in AML are awaited.

Apart from p53 reactivation, targeting immune evasion is another therapeutic strategy in TP53 mutated AML. CD47 (also known as integrin-associated protein) is a key macrophage immune checkpoint and serves as an anti-phagocytic or "do not eat me" signal [46]. CD47 is overexpressed in myeloid malignancies and its expression is enriched in AML leukaemic stem cells (LSCs) in contrast to their non-malignant counterparts [46,47]. Magrolimab (Hu5F9-G4) is a novel IgG4 antibody that blocks CD47 and induces tumor phagocytosis. Azacitidine synergizes with magrolimab by inducing pro-phagocytic "eat me" signals on leukemic blasts, thus enhancing anti-leukaemic activity [46]. Combination therapy using azacitidine and magrolimab in MDS and AML in Phase Ib study has shown encouraging efficacy. particularly in the TP53-mutant AML, a treatment-refractory group (ORR 64\%, CR 41\%) [48]. Follow-up results from ongoing expansion cohorts in AML (NCT03248479) are awaited, and a phase III trial evaluating azacitidine and magrolimab in untreated TP53-mutant AML (NCT04778397) is underway to thoroughly evaluate its therapeutic value to augment remission in TP53-mutant AML.

\subsection{KMT2A/NPM1}

KMT2A gene is located on Ch 11q23.3, with $>100$ fusion partners identified to cause leukemogenesis. Fusion genes induce aberrant HOXA and MEIS expressions secondary to the recruitment of chromatin-associated complexes, including the enzymes menin and DOT1L, resulting in stem cell-like gene expression signatures [49,50]. Recurrent chromosomal translocations involving the KMT2A gene initiate aggressive forms of leukemias and are often refractory to conventional treatments with a dismal prognosis [49]. AML with NPM1 mutation is also known to share similar stem cell-like gene expression with deregulated HOXA and MEIS genes; therefore, they are also sensitive to chromatin complex inhibition [51].

Novel drug discoveries including DOT1L inhibitors, bromodomain inhibitors, and menin inhibitors have demonstrated preclinical efficacy with ongoing validation in prospective clinical studies $[50,52,53]$. The DOT1L inhibitor pinometostat showed a modest antileukaemic activity in patients with KMT2A-rearranged leukemia in a phase I study [53]. More recently MLL-menin binding inhibitors have entered clinical testing as single agents for AML (K0539, NCT04067336 and SNDX-5613, NCT04065399), with great potential to emerge as important targeted therapies in NPM1-mutated and KMT2A-rearranged AML (Figure 1). The preliminary activity of SNDX-5613 in Phase 1 study has been reported with an ORR $48 \%$ in heavily pretreated patients harboring an MLL rearrangement or NPM1 mutation [54]. Like other novel agents, the combination of MLL-menin inhibitors with other drugs, including cytotoxic chemotherapy or novel agents, to augment treatment efficacy can potentially be explored.

\subsection{Hedgehog/Glioma-Associated Oncogene Homolog (HH-GLI) Signalling Pathway}

The Aberrant Hedgehog $(\mathrm{Hh})$ signalling pathway affects the proliferation of leukaemia stem cells, and its upregulation results in chemo-resistance in AML cell lines [55]. This pathway is regulated by the negative regulator patched $(\mathrm{PTCH})$ and positive regulator smoothened (SMO) [56]. Glasdegib acts as an oral inhibitor of the Hh pathway by 
interacting with SMO. Its proposed therapeutic potential lies in its suppression of the chemo-resistant stem cell population; hence, clinical relapse can be delayed by inhibiting this pathway. In a randomized phase 2 study of the administration of $20 \mathrm{mg}$ of LDAC subcutaneously twice daily for 10 days with or without glasdegib at $100 \mathrm{mg}$ a day in newly diagnosed AML or high-risk MDS patients, the combination of LDAC and glasdegib was shown to improve OS (8.3 months vs. 4.3 months, HR $0.51 ; 80 \% \mathrm{CI}, 0.39-0.67$, $p=0.0004)$ and CR (15\% vs. $2.3 \%$ ) [57]. This study resulted in the FDA approval of glasdegib when used in combination with LDAC. A small retrospective single-centre review using LDAC and glasdegib in R/R AML, including a 29\% relapse of AML post alloHSCT and a $45 \%$ venetoclax-treated population, showed a modest CRc of $21 \%$ with a median OS of 3.9 months [58]. More robust results from phase 3 randomized studies are awaited to definitively demonstrate the superiority of glasdegib in combination with LDAC (Table 1). The widespread adoption of the LDAC and glasdegib combination in the real world remains uncertain, considering the better response rates demonstrated in other more active combinations-for instance, HMA or LDAC plus venetoclax in the chemotherapy-unfit population. This treatment can be considered in combination with other cytotoxic therapies in the venetoclax-refractory population; however, further data are required.

\section{Immunotherapy}

Immunotherapy, including alloHSCT and donor lymphocyte infusion, has been used in the treatment of AML for many years, with $\mathrm{T}$ cells considered to be the major contributor to the success of this therapy [59]. However, the desirable graft-versus-leukaemia effect of alloHSCT is often offset by the significant and long-term side effects of graft-versus-host disease, restricting the widespread use of alloHSCT to achieve cure in AML. To date, various strategies aiming to harness the robust anti-tumour activity of $\mathrm{T}$ cells while limiting T-cell cytotoxicity against healthy tissues have been successful in the treatment of hematological malignancies, mainly B cell disorders. Nevertheless, targeted immunotherapy in AML is challenged by the lack of AML-specific target antigens and the complex clonal architecture, with multiple driver mutations existing in the AML tumour microenvironment $[60,61]$.

\subsection{Antibody-Drug Conjugates (ADCs)}

ADCs consist of three main components: a monoclonal antibody $(\mathrm{mAb})$ that recognizes an antigen target on tumor cells; a cytotoxic molecule, often referred to as a payload, and a chemical linker that connects the $\mathrm{mAb}$ and payload [62]. They are designed to bind selectively to antigen-positive cells, enabling the targeted delivery of cytotoxic payload to tumor cells.

CD33 is a transmembrane receptor expressed by committed myeloid cells, including the majority of leukemic blasts but not normal hematopoietic stem cells [63]. Hence, CD33 is an attractive target in the treatment of AML. Gemtuzumab ozogamicin (GO) is a humanized anti-CD33 conjugated to a calicheamicin derivative; once released intracellularly, it leads to DNA binding and cell death [63]. The combination of GO and chemotherapy has been shown to improve RFS and OS, especially in patients with favorable-risk core-binding factor AML and intermediate-risk disease, including NPM1-mutated AML, but not in patients with adverse-risk disease, likely related to the high levels of CD33 expression [64-66]. The addition of GO to intensive chemotherapy in NPM1-mutated AML has been shown to lead to better clearance of the NPM1 transcript level, resulting in a lower relapse rate (4-yr CIR $29.3 \%$ vs. $45.7 \%, p=0.009$ ) [64]. Overall, the prolongation of EFS is attributed to the depth of response and the prolongation of $\mathrm{CR}$ with the addition of GO [66]. The addition of GO represents a worthwhile option in the frontline setting for CD33-expressing disease $[66,67]$. However, the use of GO requires prompt recognition and management due to the increased risk of hepatic veno-occlusive disease/sinusoidal obstruction syndrome (VOD/SOS), especially following hematopoietic stem cell transplantation, prolonged thrombocytopenia, infusion-related reaction, and tumor lysis syndrome. 


\subsection{Bispecific Antibodies}

Bispecific antibodies are recombinant protein constructs that engage $\mathrm{T}$ cells through CD3 and bind to tumor-associated antigen, typically with a higher affinity. In AML, potential therapeutic targets are lineage-restricted antigens including CD33, CD123, CLL-1, and FLT3, for which early phase clinical trials are underway.

CD123 (also known as interleukin 3 receptor $\alpha$-chain, or IL3RA) is expressed on normal haematopoietic stem/progenitor cells, but it is expressed more on AML blasts and LSCs [68]. It is an attractive therapeutic target in $\mathrm{AML}$, as high CD123 expression on blasts confers poor outcomes and high CD123 expression is also enriched in primary induction failure/earlyrelapse AML [68-70]. The most advanced CD123-based immunotherapy currently in clinical development is flotetuzumab, a CD123 x CD3 dual-affinity retargeting (DART) antibody. Flotetuzumab has shown preliminary efficacy in R/R AML (NCT02152956), with a CR/CRh of $20 \%$ and a satisfactory safety profile [71]. It is of note that clinical activity was mainly observed in patients with primary induction failure (PIF) or early relapse $<6$ months (ER), a chemo-refractory group showing a CR/CRh of $27 \%$ and an ORR of $30 \%$, with a median OS of 10.2 months and 6- and 12-month survival rates of $75 \%$ and $50 \%$, respectively [71]. This is encouraging compared to the dismal outcome with an ORR 12\% for salvage chemotherapy in patients with PIF/ER and a median OS of 3 months [72,73].

The observed response of patients with PIF/ER AML to CD123-targeted immunotherapy is in accordance with the finding that an immune-infiltrated (IFN- $\gamma$-dominant) tumour microenvironment can identify patients who are less likely to respond to cytotoxic chemotherapy but more likely to respond to immunotherapy [74]. In comparison to B-cell immunotherapies, the incidence and severity of infusion-related reaction (IRR)/cytokine release syndrome (CRS) using CD123-targeted immunotherapy may be more pronounced ( $96 \%$ IRR/CRS but only $8 \%$ Grade $3-4$ ) due to the shared target antigen expression of monocytes and macrophages, which mediate IL-6 production [71]. Stepwise dosing, premedication with dexamethasone, the prompt use of tocilizumab, and temporary dose reductions or interruptions have been shown to prevent severe IRR/CRS [71]. In summary, immunotherapy targeting CD123/CD3 may offer a novel treatment option in PIF/ER AML. The expression of CD123 by leukemic stem cells further strengthens the potential therapeutic value of this target $[70,75]$.

Several potential biomarkers used to predict responders in CD123-targeted immunotherapy have been identified, including a higher CD123 receptor density and higher CD123 mRNA using gene expression profiling in patients with PIF/ER [71]. Future studies on measurable residual disease and/or leukemic stem cell eradication, as well as its impact on survival outcome using immunotherapy, are warranted in order to allow its incorporation as a frontline treatment.

T-cell-directed therapy using bispecific antibodies is a promising immunologic approach in the treatment of AML. However, the most suitable and appropriate epitopes for limiting on-target off-tumor toxicity in AML are yet to be identified. One of the proposed ways to optimize the success of bispecific antibodies in AML is to test it earlier in the treatment sequence - for instance, in the first salvage or in the MRD setting - to minimize T-cell exhaustion, as preserved T-cell function is critical for the activity of bispecific antibodies [61]. To improve survival outcomes and ensure the safe delivery of immunotherapy, ongoing efforts to determine biomarkers that will help identify the patients most likely to benefit from immunotherapy, to determine the ideal timing of therapy (e.g., frontline, MRD positive, maintenance, early salvage), and to determine the optimal combination partners and/or sequence are vital [61].

\subsection{Chimeric Antigen Receptor (CAR) T Cell Therapy}

Unlike the successes of CAR-T cell therapy in B cell malignancies, the progress of the use of CAR-T cells in the treatment of AML has been hindered, mainly due to the lack of suitable targetable antigens and the poorly tolerated consequences of complete myeloid progeny ablation [76,77]. Most of the AML antigens targeted by CAR-T cells are frequently 
expressed in normal hematopoietic stem/progenitor cells and other organs, including the lungs and liver, causing an increased risk of on-target off-tumor toxicity [61]. Early-phase AML CAR-T and CAR NK clinical trials targeting CD33, CD123, and NKG2D are ongoing, with creative solutions that actively seek to overcome therapeutic obstacles [61]. The impact of CAR-T therapy in AML is awaited with cautious optimism.

\section{Conclusions}

The powerful diagnostic technologies created in recent years as part of the therapeutic revolution have transformed the paradigm of AML treatment, with increasing focus now placed on precision medicine approaches. Targeting the disease heterogeneity of AML has provided a more rational therapeutic approach compared to the 'one-size-fits-all' approach using conventional chemotherapy. It is likely that, in the long term, targeted agents will be used in combination with chemotherapy to eliminate measurable residual disease and for maintenance treatment. The potential areas of focus for future research in AML therapy are summarized in Table 2, with specific areas for each molecular aberrations highlighted and discussed throughout the paper. The ongoing challenges will be to predict individualized treatment responses using biomarkers as well as to translate the initial responses into deep and durable disease control by incorporating these novel agents into upfront treatment.

Table 2. Potential areas for future research.

\begin{tabular}{|c|c|}
\hline Target/Therapy & Areas of Focus for Future and Ongoing Research \\
\hline FLT3 & $\begin{array}{l}\text { - } \quad \text { Role of 2nd-generation inhibitors in the front-line setting. } \\
\text { - } \quad \text { Identifying an effective combination with other novel agents. } \\
\text { - } \quad \text { Role of maintenance therapy post chemotherapy and post allogeneic transplant. } \\
\text { - } \quad \text { Potential for targeted therapies to eliminate MRD. } \\
\text { - }\end{array}$ \\
\hline IDH1/2 & $\begin{array}{l}\text { - } \quad \text { Role of IDH inhibitors in combination with frontline intensive chemotherapy. } \\
\text { - } \quad \text { Identifying optimal combinations with novel agents. } \\
\text { - } \quad \text { Describing the biomarkers of response and survival-does mutation clearance have an impact? } \\
\text { - } \quad \text { Establishing the role of IDH inhibitors in the maintenance setting. }\end{array}$ \\
\hline p53 and KMT2A & - $\quad$ Identifying effective targeted agent and combinations. \\
\hline Immunotherapy & $\begin{array}{l}\text { - } \quad \text { Single versus combination therapy. } \\
\text { - } \quad \text { Defining the role in the initial treatment of AML. } \\
\text { - } \quad \text { Establishing the role in maintenance and MRD elimination. }\end{array}$ \\
\hline
\end{tabular}

Author Contributions: Manuscript writing: all authors. Final approval of manuscript: all authors. Accountable for all aspects of the work: all authors. All authors have read and agreed to the published version of the manuscript.

Funding: This research received no external funding.

Institutional Review Board Statement: Not applicable.

Informed Consent Statement: Not applicable.

Data Availability Statement: Not applicable.

Conflicts of Interest: The authors declare no conflict of interest.

\section{References}

1. Bohl, S.R.; Bullinger, L.; Rücker, F.G. New targeted agents in acute myeloid leukemia: New hope on the rise. Int. J. Mol. Sci. 2019, 20, 1983. [CrossRef]

2. Stone, R.M.; Mandrekar, S.J.; Sanford, B.L.; Laumann, K.; Geyer, S.; Bloomfield, C.D.; Thiede, C.; Prior, T.W.; Döhner, K.; Marcucci, G.; et al. Midostaurin plus chemotherapy for acute myeloid leukemia with a FLT3 mutation. N. Engl. J. Med. 2017, 377, 454-464. [CrossRef]

3. Biorender. Available online: https://app.biorender.com/ (accessed on 26 September 2021). 
4. Perl, A.E.; Martinelli, G.; Cortes, J.E.; Neubauer, A.; Berman, E.; Paolini, S.; Montesinos, P.; Baer, M.R.; Larson, R.A.; Ustun, C.; et al. Gilteritinib or chemotherapy for relapsed or refractory FLT3-mutated AML. N. Engl. J. Med. 2019, 381, 1728-1740. [CrossRef]

5. Sasaki, K.; Kantarjian, H.M.; Kadia, T.; Patel, K.; Loghavi, S.; Garcia-Manero, G.; Jabbour, E.J.; DiNardo, C.; Pemmaraju, N.; Daver, N.; et al. Sorafenib plus intensive chemotherapy improves survival in patients with newly diagnosed, FLT3-internal tandem duplication mutation-positive acute myeloid leukemia. Cancer 2019, 125, 3755-3766. [CrossRef] [PubMed]

6. Röllig, C.; Serve, H.; Hüttmann, A.; Noppeney, R.; Müller-Tidow, C.; Krug, U.; Baldus, C.D.; Brandts, C.H.; Kunzmann, V.; Einsele, $\mathrm{H}$; ; et al. Addition of sorafenib versus placebo to standard therapy in patients aged 60 years or younger with newly diagnosed acute myeloid leukaemia (SORAML): A multicentre, phase 2, randomised controlled trial. Lancet Oncol. 2015, 16, 1691-1699. [CrossRef]

7. Wei, A.H.; Kennedy, G.A.; Morris, K.L.; Grigg, A.; He, S.; Schwarer, A.; Ting, S.B.; Enjeti, A.K.; Yuen, S.; D’Rozario, J.; et al. Results of a Phase 2, Randomized, Double-Blind Study of Sorafenib Versus Placebo in Combination with Intensive Chemotherapy in Previously Untreated Patients with FLT3-ITD Acute Myeloid Leukemia (ALLG AMLM16). 2020. Available online: https://ash.confex.com/ash/2020/webprogram/Paper137334.html (accessed on 1 March 2021).

8. Ravandi, F.; Yi, C.A.; Cortes, J.; Levis, M.; Faderl, S.; Garcia-Manero, G.; Jabbour, E.; Konopleva, M.; O’Brien, S.; Estrov, Z.; et al Final report of phase II study of sorafenib, cytarabine and idarubicin for initial therapy in younger patients with acute myeloid leukemia. Leukemia 2014, 28, 1543-1545. [CrossRef]

9. Pratz, K.W.; Cherry, M.; Altman, J.K.; Cooper, B.W.; Cruz, J.C.; Jurcic, J.G.; Levis, M.; Lin, T.; Perl, A.E.; Podoltsev, N.A.; et al. A phase 1 study of gilteritinib in combination with induction and consolidation chemotherapy in patients with newly diagnosed AML: Final results. Blood 2020, 136, 16-17. [CrossRef]

10. Esteve, J.; Schots, R.; Del Castillo, T.B.; Lee, J.-H.; Wang, E.S.; Dinner, S.; Minden, M.D.; Salamero, O.; Sierra, J.; Yoshimoto, G.; et al. Multicenter, open-label, 3-arm study of gilteritinib, gilteritinib plus azacitidine, or azacitidine alone in newly diagnosed FLT3 mutated (FLT3mut+) acute myeloid leukemia (AML) patients ineligible for intensive induction chemotherapy: Findings from the safety cohort. Blood 2018, 132, 2736. [CrossRef]

11. Döhner, K.; Thiede, C.; Jahn, N.; Panina, E.; Gambietz, A.; Larson, R.A.; Prior, T.W.; Marcucci, G.; Jones, D.; Krauter, J.; et al. Impact of NPM1/FLT3-ITD genotypes defined by the 2017 European LeukemiaNet in patients with acute myeloid leukemia. Blood 2020, 135, 371-380. [CrossRef]

12. Röllig, C.; Serve, H.; Noppeney, R.; Hanoun, M.; Krug, U.; Baldus, C.D.; Brandts, C.H.; Kunzmann, V.; Einsele, H.; Krämer, A.; et al. Sorafenib or placebo in patients with newly diagnosed acute myeloid leukaemia: Long-term follow-up of the randomized controlled SORAML trial. Leukemia 2021, 35, 2517-2525. [CrossRef]

13. Schlenk, R.F.; Weber, D.; Fiedler, W.; Salih, H.R.; Wulf, G.; Salwender, H.; Schroeder, T.; Kindler, T.; Lübbert, M.; Wolf, D.; et al. Midostaurin added to chemotherapy and continued single-agent maintenance therapy in acute myeloid leukemia with FLT3-ITD. Blood 2019, 133, 840-851. [CrossRef] [PubMed]

14. Schmalbrock, L.K.; Dolnik, A.; Cocciardi, S.; Sträng, E.; Theis, F.; Jahn, N.; Panina, E.; Blätte, T.J.; Herzig, J.; Skambraks, S.; et al. Clonal evolution of acute myeloid leukemia with FLT3-ITD mutation under treatment with midostaurin. Blood 2021, 137, 3093-3104. [CrossRef] [PubMed]

15. Daver, N.; Altman, J.K.; Maly, J.; Levis, M.; Ritchie, E.; Litzow, M.; McCloskey, J.K.; Smith, C.C.; Schiller, G.J.; Bradley, T.; et al. Efficacy and safety of venetoclax in combination with gilteritinib for relapsed/refractory FLT3-mutated acute myeloid leukemia: Updated analyses of a Phase 1b study. EHA 2021. Blood 2021, 136, 20-22. [CrossRef]

16. Xuan, L.; Wang, Y.; Huang, F.; Fan, Z.; Xu, Y.; Sun, J.; Xu, N.; Deng, L.; Li, X.; Liang, X.; et al. Sorafenib maintenance in patients with FLT3-ITD acute myeloid leukaemia undergoing allogeneic haematopoietic stem-cell transplantation: An open-label, multicentre, randomised phase 3 trial. Lancet Oncol. 2020, 21, 1201-1212. [CrossRef]

17. Burchert, A.; Bug, G.; Fritz, L.V.; Finke, J.; Stelljes, M.; Röllig, C.; Wollmer, E.; Wäsch, R.; Bornhäuser, M.; Berg, T.; et al. Sorafenib maintenance after allogeneic hematopoietic stem cell transplantation for acute myeloid leukemia with FLT3-internal tandem duplication mutation (SORMAIN). J. Clin. Oncol. 2020, 38, JCO1903345. [CrossRef]

18. Gagelmann, N.; Wolschke, C.; Klyuchnikov, E.; Christopeit, M.; Ayuk, F.; Kröger, N. TKI Maintenance after stem-cell transplantation for FLT3-itd positive acute myeloid leukemia: A systematic review and meta-analysis. Front. Immunol. 2021, 12. [CrossRef]

19. Figueroa, M.E.; Abdel-Wahab, O.; Lu, C.; Ward, P.S.; Patel, J.; Shih, A.; Li, Y.; Bhagwat, N.; Vasantha Kumar, A.; Fernandez, H.F.; et al. Leukemic IDH1 and IDH2 mutations result in a hypermethylation phenotype, disrupt TET2 function, and impair hematopoietic differentiation. Cancer Cell 2010, 18, 553-567. [CrossRef]

20. Ward, P.; Patel, J.; Wise, D.; Abdel-Wahab, O.; Bennett, B.D.; Coller, H.A.; Cross, J.; Fantin, V.R.; Hedvat, C.; Perl, A.E.; et al. The common feature of leukemia-associated IDH1 and IDH2 mutations is a neomorphic enzyme activity converting $\alpha$-ketoglutarate to 2-hydroxyglutarate. Cancer Cell 2010, 17, 225-234. [CrossRef]

21. Lu, C.; Ward, P.S.; Kapoor, G.S.; Rohle, D.; Turcan, S.; Abdel-Wahab, O.; Edwards, C.R.; Khanin, R.; Figueroa, M.E.; Melnick, A.; et al. IDH mutation impairs histone demethylation and results in a block to cell differentiation. Nature 2012, 483, 474-478. [CrossRef]

22. Stein, E.M.; Dinardo, C.D.; Pollyea, D.A.; Fathi, A.T.; Roboz, G.J.; Altman, J.K.; Stone, R.M.; DeAngelo, D.J.; Levine, R.L.; Flinn, I.W.; et al. Enasidenib in mutant IDH2 relapsed or refractory acute myeloid leukemia. Blood 2017, 130, 722-731. [CrossRef] [PubMed] 
23. Dinardo, C.D.; Stein, E.M.; De Botton, S.; Roboz, G.J.; Altman, J.K.; Mims, A.S.; Swords, R.; Collins, R.H.; Mannis, G.N.; Pollyea, D.A.; et al. Durable remissions with ivosidenib in IDH1-mutated relapsed or refractory AML. N. Engl. J. Med. 2018, 378, 2386-2398. [CrossRef] [PubMed]

24. Roboz, G.J.; Dinardo, C.D.; Stein, E.M.; De Botton, S.; Mims, A.S.; Prince, G.T.; Altman, J.K.; Arellano, M.L.; Donnellan, W.; Erba, H.P.; et al. Ivosidenib induces deep durable remissions in patients with newly diagnosed IDH1-mutant acute myeloid leukemia. Blood 2020, 135, 463-471. [CrossRef] [PubMed]

25. Pollyea, D.A.; Tallman, M.S.; De Botton, S.; Kantarjian, H.M.; Collins, R.; Stein, A.S.; Frattini, M.G.; Xu, Q.; Tosolini, A.; See, W.L.; et al. Enasidenib, an inhibitor of mutant IDH2 proteins, induces durable remissions in older patients with newly diagnosed acute myeloid leukemia. Leukemia 2019, 33, 2575-2584. [CrossRef] [PubMed]

26. Döhner, H.; Dolnik, A.; Tang, L.; Seymour, J.F.; Minden, M.D.; Stone, R.M.; Del Castillo, T.B.; Al-Ali, H.K.; Santini, V.; Vyas, P.; et al. Cytogenetics and gene mutations influence survival in older patients with acute myeloid leukemia treated with azacitidine or conventional care. Leukemia 2018, 32, 2546-2557. [CrossRef] [PubMed]

27. Dombret, H.; Seymour, J.F.; Butrym, A.; Wierzbowska, A.; Selleslag, D.; Jang, J.H.; Kumar, R.; Cavenagh, J.; Schuh, A.C.; Candoni, A.; et al. International phase 3 study of azacitidine vs. conventional care regimens in older patients with newly diagnosed AML with $>30 \%$ blasts. Blood 2015, 126, 291-299. [CrossRef] [PubMed]

28. DiNardo, C.; Montesinos, P.; Schuh, A.; Papayannidis, C.; Vyas, P.; Wei, A.; Ommen, H.; Semochkin, S.; Kim, H.; Larson, R.; et al. A Phase 3 Study of Enasidenib Versus Conventional Care Regimens in Older Patients with Late-Stage Mutant IDH2 Relapsed/ Refractory Acute Myeloid Leukemia. 2021. Available online: https:/ /ibrary.ehaweb.org/eha/2021/eha2021-virtual-congress/ 325217 (accessed on 26 September 2021).

29. Dinardo, C.D.; Schuh, A.C.; Stein, E.M.; Fernandez, P.M.; Wei, A.; De Botton, S.; Zeidan, A.M.; Fathi, A.T.; Quek, L.; Kantarjian, H.M.; et al. Enasidenib plus azacitidine significantly improves complete remission and overall response compared with azacitidine alone in patients with newly diagnosed acute myeloid leukemia (AML) with isocitrate dehydrogenase 2 (IDH2) mutations: Interim phase II results from an ongoing, randomized study. Blood 2019, 134, 643. [CrossRef]

30. Dinardo, C.D.; Stein, A.S.; Stein, E.M.; Fathi, A.T.; Frankfurt, O.; Schuh, A.C.; Döhner, H.; Martinelli, G.; Patel, P.A.; Raffoux, E.; et al. Mutant isocitrate dehydrogenase 1 inhibitor ivosidenib in combination with azacitidine for newly diagnosed acute myeloid leukemia. J. Clin. Oncol. 2021, 39, 57-65. [CrossRef]

31. Montesinos, P.; Recher, C.; Zarzycka, E.; Doronin, V.; McCulloch, D.; Polo, S.V.; Calado, R.T.; Jang, J.H.; Miyazaki, Y.; Wang, J.; et al. Agile: Phase 3, double-blind, randomized, placebo-controlled study of ivosidenib in combination with azacitidine in adults with newly diagnosed acute myeloid leukemia and an IDH1 mutation. Blood 2019, 134, 2593.

32. Stein, E.M.; Dinardo, C.D.; Fathi, A.T.; Pollyea, D.A.; Stone, R.M.; Altman, J.K.; Roboz, G.J.; Patel, M.R.; Collins, R.; Flinn, I.W.; et al. Molecular remission and response patterns in patients with mutant-IDH2 acute myeloid leukemia treated with enasidenib. Blood 2019, 133, 676-687. [CrossRef]

33. Stein, E.M.; DiNardo, C.D.; Fathi, A.T.; Mims, A.S.; Pratz, K.W.; Savona, M.R.; Stein, A.S.; Stone, R.M.; Winer, E.S.; Seet, C.S.; et al. Ivosidenib or enasidenib combined with intensive chemotherapy in patients with newly diagnosed AML: A phase 1 study. Blood 2021, 137, 1792-1803. [CrossRef]

34. Choe, S.; Wang, H.; Dinardo, C.D.; Stein, E.M.; De Botton, S.; Roboz, G.J.; Altman, J.K.; Mims, A.S.; Watts, J.M.; Pollyea, D.A.; et al. Molecular mechanisms mediating relapse following ivosidenib monotherapy in IDH1-mutant relapsed or refractory AML. Blood Adv. 2020, 4, 1894-1905. [CrossRef]

35. Intlekofer, A.; Shih, A.H.; Wang, B.; Nazir, A.; Rustenburg, A.S.; Albanese, S.; Patel, M.; Famulare, C.; Correa, F.M.; Takemoto, N.; et al. Acquired resistance to IDH inhibition through trans or cis dimer-interface mutations. Nat. Cell Biol. 2018, 559, 125-129. [CrossRef]

36. Duchmann, M.; Micol, J.-B.; Duployez, N.; Raffoux, E.; Thomas, X.; Marolleau, J.-P.; Braun, T.; Adès, L.; Chantepie, S.P.; Lemasle, E.; et al. Prognostic significance of concurrent gene mutations in intensively treated patients with IDH-mutated AML, an ALFA study. Blood 2021, 137, 2827-2837. [CrossRef]

37. Chan, S.M.; Thomas, D.; Corces-Zimmerman, M.R.; Xavy, S.; Rastogi, S.; Hong, W.-J.; Zhao, F.; Medeiros, B.C.; Tyvoll, D.A.; Majeti, R. Isocitrate dehydrogenase 1 and 2 mutations induce BCL-2 dependence in acute myeloid leukemia. Nat. Med. 2015, 21, 178-184. [CrossRef]

38. Dinardo, C.D.; Jonas, B.A.; Pullarkat, V.; Thirman, M.J.; Garcia, J.S.; Wei, A.H.; Konopleva, M.; Döhner, H.; Letai, A.; Fenaux, P.; et al. Azacitidine and venetoclax in previously untreated acute myeloid leukemia. N. Engl. J. Med. 2020, 383, 617-629. [CrossRef]

39. Kadia, T.M.; Jain, P.; Ravandi, F.; Garcia-Manero, G.; Andreef, M.; Takahashi, K.; Borthakur, G.; Jabbour, E.; Konopleva, M.; Daver, N.G.; et al. TP53 mutations in newly diagnosed acute myeloid leukemia: Clinicomolecular characteristics, response to therapy, and outcomes. Cancer 2016, 122, 3484-3491. [CrossRef]

40. Rücker, F.G.; Schlenk, R.F.; Bullinger, L.; Kayser, S.; Teleanu, V.; Kett, H.; Habdank, M.; Kugler, C.-M.; Holzmann, K.; Gaidzik, V.I.; et al. TP53 alterations in acute myeloid leukemia with complex karyotype correlate with specific copy number alterations, monosomal karyotype, and dismal outcome. Blood 2012, 119, 2114-2121. [CrossRef]

41. Boettcher, S.; Miller, P.G.; Sharma, R.; McConkey, M.; Leventhal, M.; Krivtsov, A.V.; Giacomelli, A.O.; Wong, W.; Kim, J.; Chao, S.; et al. A dominant-negative effect drives selection of TP53 missense mutations in myeloid malignancies. Science 2019, 365, 599-604. [CrossRef] 
42. Zhang, Q.; Bykov, V.J.N.; Wiman, K.G.; Zawacka-Pankau, J. APR-246 reactivates mutant p53 by targeting cysteines 124 and 277. Cell Death Dis. 2018, 9, 1-12. [CrossRef] [PubMed]

43. Bykov, V.J.N.; Eriksson, S.E.; Bianchi, J.; Wiman, K. Targeting mutant p53 for efficient cancer therapy. Nat. Rev. Cancer 2018, 18, 89-102. [CrossRef] [PubMed]

44. Sallman, D.A.; DeZern, A.E.; Garcia-Manero, G.; Steensma, D.P.; Roboz, G.J.; Sekeres, M.A.; Cluzeau, T.; Sweet, K.L.; McLemore, A.; McGraw, K.L.; et al. Eprenetapopt (APR-246) and azacitidine in TP53-mutant myelodysplastic syndromes. J. Clin. Oncol. 2021, 39, 1584-1594. [CrossRef]

45. Cluzeau, T.; Sebert, M.; Rahmé, R.; Cuzzubbo, S.; Lehmann-Che, J.; Madelaine, I.; Peterlin, P.; Bève, B.; Attalah, H.; Chermat, F.; et al. Eprenetapopt plus azacitidine in TP53-mutated myelodysplastic syndromes and acute myeloid leukemia: A phase II study by the groupe francophone des myélodysplasies (GFM). J. Clin. Oncol. 2021, 39, 1575-1583. [CrossRef] [PubMed]

46. Chao, M.P.; Takimoto, C.H.; Feng, D.D.; McKenna, K.; Gip, P.; Liu, J.; Volkmer, J.-P.; Weissman, I.L.; Majeti, R. Therapeutic targeting of the macrophage immune checkpoint CD47 in myeloid malignancies. Front. Oncol. 2020, 9, 1380. [CrossRef] [PubMed]

47. Jaiswal, S.; Jamieson, C.H.; Pang, W.W.; Park, C.Y.; Chao, M.P.; Majeti, R.; Traver, D.; Van Rooijen, N.; Weissman, I.L. CD47 is upregulated on circulating hematopoietic stem cells and leukemia cells to avoid phagocytosis. Cell 2009, 138, 271-285. [CrossRef] [PubMed]

48. Sallman, D.; Asch, A.; Kambhampati, S.; Al Malki, M.; Zeidner, J.; Donnellan, W.; Lee, D.; Vyas, P.; Jeyakumar, D.; Mannis, G.; et al. AML-196: The first-in-class anti-CD47 antibody magrolimab in combination with azacitidine is well tolerated and effective in AML patients: Phase 1b results. Clin. Lymphoma Myeloma Leuk. 2021, 21, S290. [CrossRef]

49. Krivtsov, A.V.; Armstrong, S.A. MLL translocations, histone modifications and leukaemia stem-cell development. Nat. Rev. Cancer 2007, 7, 823-833. [CrossRef]

50. Krivtsov, A.V.; Evans, K.; Gadrey, J.Y.; Eschle, B.K.; Hatton, C.; Uckelmann, H.J.; Ross, K.N.; Perner, F.; Olsen, S.N.; Pritchard, T.; et al. A menin-MLL inhibitor induces specific chromatin changes and eradicates disease in models of MLL-rearranged leukemia. Cancer Cell 2019, 36, 660-673. [CrossRef]

51. Kühn, M.W.M.; Song, E.; Feng, Z.; Sinha, A.; Chen, C.-W.; Deshpande, A.J.; Cusan, M.; Farnoud, N.; Mupo, A.; Grove, C.; et al. Targeting chromatin regulators inhibits leukemogenic gene expression in NPM1 mutant leukemia. Cancer Discov. 2016, 6, 1166-1181. [CrossRef]

52. Dawson, M.A.; Prinjha, R.K.; Dittmann, A.; Giotopoulos, G.; Bantscheff, M.; Chan, W.-I.; Robson, S.C.; Chung, C.-W.; Hopf, C.; Savitski, M.M.; et al. Inhibition of BET recruitment to chromatin as an effective treatment for MLL-fusion leukaemia. Nat. Cell Biol. 2011, 478, 529-533. [CrossRef]

53. Stein, E.M.; Garcia-Manero, G.; Rizzieri, D.A.; Tibes, R.; Berdeja, J.G.; Savona, M.R.; Jongen-Lavrenic, M.; Altman, J.K.; Thomson, B.; Blakemore, S.J.; et al. The DOT1L inhibitor pinometostat reduces H3K79 methylation and has modest clinical activity in adult acute leukemia. Blood 2018, 131, 2661-2669. [CrossRef]

54. Syndax Pharmaceuticals Announces Preclinical Profile and Initial Phase 1 Data Demonstrating Clinical Activity of Menin Inhibitor SNDX-5613 in Adults with Relapsed/Refractory Acute Leukemias. Available online: https://www.biospace.com/article/ releases/syndax-pharmaceuticals-announces-preclinical-profile-and-initial-phase-1-data-demonstrating-clinical-activity-ofmenin-inhibitor-sndx-5613-in-adults-with-relapsed-refractory-acute-leukemias/ (accessed on 27 April 2020).

55. Queiroz, K.C.S.; Ruela-De-Sousa, R.R.; Fuhler, G.M.; Aberson, H.L.; Ferreira, C.V.; Peppelenbosch, M.; Spek, C.A. Hedgehog signaling maintains chemoresistance in myeloid leukemic cells. Oncogene 2010, 29, 6314-6322. [CrossRef]

56. Irvine, D.A.; Copland, M. Targeting hedgehog in hematologic malignancy. Blood 2012, 119, 2196-2204. [CrossRef]

57. Cortes, J.E.; Heidel, F.H.; Hellmann, A.; Fiedler, W.; Smith, B.D.; Robak, T.; Montesinos, P.; Pollyea, D.A.; DesJardins, P.; Ottmann, O; ; et al. Randomized comparison of low dose cytarabine with or without glasdegib in patients with newly diagnosed acute myeloid leukemia or high-risk myelodysplastic syndrome. Leukemia 2019, 33, 379-389. [CrossRef]

58. Zucenka, A.; Maneikis, K.; Pugaciute, B.; Ringeleviciute, U.; Dapkeviciute, A.; Davainis, L.; Daukelaite, G.; Burzdikaite, P.; Staras, V.; Griskevicius, L. Glasdegib in combination with low-dose Cytarabine for the outpatient treatment of relapsed or refractory acute myeloid leukemia in unfit patients. Ann. Hematol. 2021, 100, 1195-1202. [CrossRef]

59. Schmid, C.; Labopin, M.; Schaap, N.; Veelken, H.; Schleuning, M.; Stadler, M.; Finke, J.; Hurst, E.; Baron, F.; Ringden, O.; et al. Prophylactic donor lymphocyte infusion after allogeneic stem cell transplantation in acute leukaemia-a matched pair analysis by the acute leukaemia working party of EBMT. Br. J. Haematol. 2019, 184, 782-787. [CrossRef] [PubMed]

60. Haubner, S.; Perna, F.; Köhnke, T.; Schmidt, C.; Berman, S.; Augsberger, C.; Schnorfeil, F.M.; Krupka, C.; Lichtenegger, F.S.; Liu, X.; et al. Coexpression profile of leukemic stem cell markers for combinatorial targeted therapy in AML. Leukemia 2019, 33, 64-74. [CrossRef]

61. Daver, N.; Alotaibi, A.S.; Bücklein, V.; Subklewe, M. T-cell-based immunotherapy of acute myeloid leukemia: Current concepts and future developments. Leukemia 2021, 35, 1843-1863. [CrossRef] [PubMed]

62. Kovtun, Y.V.; Audette, C.A.; Ye, Y.; Xie, H.; Ruberti, M.F.; Phinney, S.J.; Leece, B.A.; Chittenden, T.; Blättler, W.A.; Goldmacher, V.S. Antibody-drug conjugates designed to eradicate tumors with homogeneous and heterogeneous expression of the target antigen. Cancer Res. 2006, 66, 3214-3221. [CrossRef] [PubMed]

63. Walter, R.B.; Appelbaum, F.R.; Estey, E.H.; Bernstein, I.D. Acute myeloid leukemia stem cells and CD33-targeted immunotherapy. Blood 2012, 119, 6198-6208. [CrossRef] 
64. Kapp-Schwoerer, S.; Weber, D.; Corbacioglu, A.; Gaidzik, V.I.; Paschka, P.; Krönke, J.; Theis, F.; Rücker, F.G.; Teleanu, M.-V.; Panina, E.; et al. Impact of gemtuzumab ozogamicin on MRD and relapse risk in patients with NPM1-mutated AML: Results from the AMLSG 09-09 trial. Blood 2020, 136, 3041-3050. [CrossRef]

65. Hills, R.K.; Castaigne, S.; Appelbaum, F.R.; Delaunay, J.; Petersdorf, S.; Othus, M.; Estey, E.H.; Dombret, H.; Chevret, S.; Ifrah, $\mathrm{N}$; ; et al. Addition of gemtuzumab ozogamicin to induction chemotherapy in adult patients with acute myeloid leukaemia: A meta-analysis of individual patient data from randomised controlled trials. Lancet Oncol. 2014, 15, 986-996. [CrossRef]

66. Lambert, J.; Pautas, C.; Terré, C.; Raffoux, E.; Turlure, P.; Caillot, D.; Legrand, O.; Thomas, X.; Gardin, C.; Gogat-Marchant, K.; et al. Gemtuzumab ozogamicin for de novo acute myeloid leukemia: Final efficacy and safety updates from the open-label, phase III ALFA-0701 trial. Haematologica 2019, 104, 113-119. [CrossRef]

67. Burnett, A.K.; Hills, R.K.; Milligan, D.; Kjeldsen, L.; Kell, J.; Russell, N.H.; Yin, J.A.; Hunter, A.; Goldstone, A.H.; Wheatley, K. Identification of patients with acute myeloblastic leukemia who benefit from the addition of gemtuzumab ozogamicin: Results of the MRC AML15 Trial. J. Clin. Oncol. 2011, 29, 369-377. [CrossRef]

68. Testa, U.; Riccioni, R.; Militi, S.; Coccia, E.M.; Stellacci, E.; Samoggia, P.; Latagliata, R.; Mariani, G.; Rossini, A.; Battistini, A.; et al. Elevated expression of IL-3R $\alpha$ in acute myelogenous leukemia is associated with enhanced blast proliferation, increased cellularity, and poor prognosis. Blood 2002, 100, 2980-2988. [CrossRef]

69. Kandeel, E.Z.; El Sharkawy, N.; Hanafi, M.; Samra, M.; Kamel, A. Tracing leukemia stem cells and their influence on clinical course of adult acute myeloid leukemia. Clin. Lymphoma Myeloma Leuk. 2020, 20, 383-393. [CrossRef] [PubMed]

70. Al-Hussaini, M.; Rettig, M.P.; Ritchey, J.K.; Karpova, D.; Uy, G.L.; Eissenberg, L.G.; Gao, F.; Eades, W.C.; Bonvini, E.; Chichili, G.R.; et al. Targeting CD123 in acute myeloid leukemia using a T-cell-directed dual-affinity retargeting platform. Blood 2016, 127, 122-131. [CrossRef] [PubMed]

71. Uy, G.L.; Aldoss, I.; Foster, M.C.; Sayre, P.H.; Wieduwilt, M.J.; Advani, A.S.; Godwin, J.E.; Arellano, M.L.; Sweet, K.L.; Emadi, A.; et al. Flotetuzumab as salvage immunotherapy for refractory acute myeloid leukemia. Blood 2021, 137, 751-762. [CrossRef]

72. Estey, E.; Kornblau, S.; Pierce, S.; Kantarjian, H.; Beran, M.; Keating, M. Relapsed or primary refractory AML—A stratification system. Blood 1996, 88, 756. [CrossRef] [PubMed]

73. Walter, R.B.; Othus, M.; Löwenberg, B.; Ossenkoppele, G.J.; Petersdorf, S.H.; Pabst, T.; Vekemans, M.-C.; Appelbaum, F.R.; Erba, H.P.; Estey, E.H. Empiric definition of eligibility criteria for clinical trials in relapsed/refractory acute myeloid leukemia: Analysis of 1,892 patients from HOVON/SAKK and SWOG. Haematologica 2015, 100, e409-e411. [CrossRef]

74. Vadakekolathu, J.; Minden, M.D.; Hood, T.; Church, S.E.; Reeder, S.; Altmann, H.; Sullivan, A.H.; Viboch, E.J.; Patel, T.; Ibrahimova, N.; et al. Immune landscapes predict chemotherapy resistance and immunotherapy response in acute myeloid leukemia. Sci. Transl. Med. 2020, 12, 546. [CrossRef]

75. Zahran, A.M.; Aly, S.S.; Rayan, A.; El-Badawy, O.; Fattah, M.A.; Ali, A.M.; ElBadre, H.M.; Hetta, H.F. Survival outcomes of CD34+CD38-LSCs and their expression of CD123 in adult AML patients. Oncotarget 2018, 9, 34056-34065. [CrossRef] [PubMed]

76. Cummins, K.; Gill, S. Chimeric antigen receptor T-cell therapy for acute myeloid leukemia: How close to reality? Haematologica 2019, 104, 1302-1308. [CrossRef] [PubMed]

77. June, C.H.; O'Connor, R.S.; Kawalekar, O.U.; Ghassemi, S.; Milone, M.C. CAR T cell immunotherapy for human cancer. Science 2018, 359, 1361-1365. [CrossRef] [PubMed] 